\title{
A note on the existence of solutions for a class of quasilinear elliptic equations: an Orlicz-Sobolev space setting
}

Yang Yang $^{1 *}$ and Jihui Zhang ${ }^{2}$

\footnotetext{
"Correspondence: yynjnu@126.com 'School of Science, Jiangnan University, Wuxi, 214122, People's Republic of China

Full list of author information is available at the end of the article
}

\section{Abstract}

In this note, we study the existence and multiplicity of solutions for the quasilinear elliptic problem as follows:

$$
\begin{cases}-\operatorname{div}(a(|\nabla u|) \nabla u)=f(x, u), & \text { in } \Omega ; \\ u=0, & \text { on } \partial \Omega,\end{cases}
$$

where $\Omega \subset R^{N}$ is a bounded domain with a smooth boundary. The existence and multiplicity of solutions are obtained by a version of the symmetric mountain pass theorem.

Keywords: Orlicz-Sobolev spaces; symmetric mountain pass theorem; quasilinear elliptic equations

\section{Introduction}

In this note, we discuss the existence and multiplicity of solutions of the following boundary value problem:

$$
\begin{cases}-\operatorname{div}(a(|\nabla u|) \nabla u)=f(x, u), & \text { in } \Omega \\ u=0, & \text { on } \partial \Omega\end{cases}
$$

where $\Omega \subset R^{N}$ is a bounded domain with a smooth boundary $\partial \Omega$. The function $a$ is such that $p: R \rightarrow R$ defined by

$$
p(t)= \begin{cases}a(|t|) t, & t \neq 0 \\ 0, & t=0\end{cases}
$$

is an increasing homeomorphism from $R$ onto itself and the continuous function $f(x, t) \in$ $C(\bar{\Omega} \times R, R)$ satisfies $f(x, 0)=0, x \in \bar{\Omega}$. Especially, when $a(t)=|t|^{p-2}$, the problem (1.1) is the well-known p-Laplacian equation. There is a large number of papers on the existence of solutions for the p-Laplacian equation. But the problem (1.1) possesses more complicated nonlinearities. For example, it is inhomogeneous and has an important physical background, e.g.,

\section{Springer}

( 2012 Yang and Zhang; licensee Springer. This is an Open Access article distributed under the terms of the Creative Commons Attribution License (http://creativecommons.org/licenses/by/2.0), which permits unrestricted use, distribution, and reproduction in any medium, provided the original work is properly cited. 
(a) nonlinear elasticity: $P(t)=\left(1+t^{2}\right)^{\gamma}-1, \gamma>\frac{1}{2}$;

(b) plasticity: $P(t)=t^{\alpha}(\log (1+t))^{\beta}, \alpha \geq 1, \beta>0$;

(c) generalized Newtonian fluids: $P(t)=\int_{0}^{t} s^{1-\alpha}\left(\sinh ^{-1} s\right)^{\beta} d s, 0 \leq \alpha \leq 1, \beta>0$.

So, in the discussions, some special techniques are needed, and the problem (1.1) has been studied in an Orlicz-Sobolev space and received considerable attention in recent years; see, for instance, the papers [1-9]. In paper [9], Fang and Tan discussed the problem (1.1) under the conditions that $f(x, t)$ was odd in $t$. They got the first result that when $h^{+}<p^{-}$, and $f(x, t) \geq C t^{q-1}$ for $0<t<\delta, q<p^{-}$, the problem (1.1) had a sequence of solutions by genus theory. The second result is that when $f(x, t)$ satisfies $0<\alpha F(x, t) \leq t f(x, t), \forall x \in \bar{\Omega}$, $t \neq 0, \alpha>p^{+}$and $f(x, t)=o(p(|t|))$ as $|t| \rightarrow 0$, the problem (1.1) has infinitely many pairs of solutions which correspond to the positive critical values by the symmetric mountain pass theorem.

Motivated by their results, in this note, we discuss the problem (1.1) when $f(x, t)$ is still odd in $t$ but it satisfies weaker conditions than [9]; and furthermore, we need not know the behaviors of $f(x, t)$ near the zero. If $h^{+}>p^{-}$, we can get multiplicity of solutions by a version of the symmetric mountain pass theorem.

The paper is organized as follows. In Section 2, we present some preliminary knowledge on the Orlicz-Sobolev spaces and give the main result. In Section 3, we make the proof.

\section{Preliminaries}

Obviously, the problem (1.1) allows a nonhomogeneous function $p$ in the differential operator defining the problem (1.1). To deal with this situation, we introduce an Orlicz-Sobolev space setting for the problem (1.1) as follows.

Let

$$
P(t)=\int_{0}^{t} p(s) d s, \quad \widetilde{P}(t)=\int_{0}^{t} p^{-1}(s) d s, \quad t \in R,
$$

then $P$ and $\widetilde{P}$ are complementary $N$-functions (see [10]), which define the Orlicz spaces $L^{P}:=L^{P}(\Omega)$ and $L^{\widetilde{P}}:=L^{\widetilde{P}}(\Omega)$ respectively.

Throughout this paper, we assume the following condition on $P$ :

$$
\text { (p) } 1<p^{-}:=\inf _{t>0} \frac{t p(t)}{P(t)} \leq p^{+}:=\sup _{t>0} \frac{t p(t)}{P(t)}<+\infty \text {. }
$$

Under the condition ( $p$ ), the Orlicz space $L^{P}$ coincides with the set (equivalence classes) of measurable functions $u: \Omega \rightarrow R$ such that

$$
\int_{\Omega} P(|u|) d x<+\infty
$$

and is equipped with the (Luxemburg) norm, i.e.,

$$
|u|_{P}:=\inf \left\{k>0: \int_{\Omega} P\left(\frac{|u|}{k}\right) d x<1\right\} .
$$

We will denote by $W^{1, P}(\Omega)$ the corresponding Orlicz-Sobolev space with the norm

$$
\|u\|_{W^{1, P}(\Omega)}:=|u|_{P}+\|\nabla u\|_{P}
$$


and define $W_{0}^{1, P}(\Omega)$ as the closure of $C_{0}^{\infty}$ in $W^{1, P}(\Omega)$. In this note, we will use the following equivalent norm on $W_{0}^{1, P}(\Omega)$ :

$$
\|u\|:=\inf \left\{k>0: \int_{\Omega} P\left(\frac{|\nabla u|}{k}\right) d x<1\right\} .
$$

Now, we introduce the Orlicz-Sobolev conjugate $P_{*}$ of $P$, which is given by

$$
P_{*}^{-1}(t):=\int_{0}^{t} \frac{p^{-1}(\tau)}{\tau^{\frac{N+1}{N}}} d \tau
$$

where we suppose that

$$
\lim _{t \rightarrow 0} \int_{t}^{1} \frac{p^{-1}(\tau)}{\tau^{\frac{N+1}{N}}} d \tau<+\infty, \quad \lim _{t \rightarrow \infty} \int_{1}^{t} \frac{p^{-1}(\tau)}{\tau^{\frac{N+1}{N}}} d \tau=+\infty
$$

Let $p_{*}^{-}:=\inf _{t>0} \frac{t P_{*}^{\prime}(t)}{P_{*}(t)}, p_{*}^{+}:=\sup _{t>0} \frac{t P_{*}^{\prime}(t)}{P_{*}(t)}$. Throughout this paper, we assume that $p^{+}<p_{*}^{-}$. Now, we will make the following assumptions on $f(x, t)$.

$\left(f_{*}\right)$ There exists an odd increasing homeomorphism $h$ from $R$ to $R$, and nonnegative constants $c_{1}, c_{2}$ such that

$$
|f(x, t)| \leq c_{1}+c_{2} h(|t|), \quad \forall t \in R, \forall x \in \bar{\Omega},
$$

and $\lim _{t \rightarrow+\infty} \frac{H(t)}{P_{*}(k t)}=0, \forall k>0$, where

$$
H(t):=\int_{0}^{t} h(s) d s
$$

Let

$$
\widetilde{H}(t):=\int_{0}^{t} h^{-1}(s) d s
$$

then we can obtain complementary $N$-functions which define corresponding Orlicz spaces $L^{H}$ and $L^{H_{*}}$.

Similar to the condition $(p)$, we also assume the following condition on $H$ :

(h) $1<h^{-}:=\inf _{t>0} \frac{t h(t)}{H(t)} \leq h^{+}:=\sup _{t>0} \frac{t h(t)}{H(t)}<+\infty$.

In order to prove our results, we now state some useful lemmas.

Lemma 2.1 [10] Under the condition $(p)$, the spaces $L^{P}(\Omega), W_{0}^{1, P}(\Omega)$ and $W^{1, P}(\Omega)$ are separable and reflexive Banach spaces.

Lemma $2.2[10]$ Under the condition $\left(f_{*}\right)$, the embedding $W_{0}^{1, P}(\Omega) \hookrightarrow L^{H}(\Omega)$ is compact.

Lemma 2.3 [2] Let $\rho(u)=\int_{\Omega} P(u) d x$, we have

(1) if $|u|_{P}<1$, then $|u|_{P}^{p^{+}} \leq \rho(u) \leq|u|_{P}^{p^{-}}$; 
(2) if $|u|_{P}>1$, then $|u|_{P}^{p^{-}} \leq \rho(u) \leq|u|_{P}^{p^{+}}$;

(3) if $0<t<1$, then $t^{p^{+}} P(u) \leq P(t u) \leq t^{p^{-}} P(u)$;

(4) if $t>1$, then $t^{p^{-}} P(u) \leq P(t u) \leq t^{p^{+}} P(u)$.

Lemma 2.4 [11-13] Let $E=V+X$, where $E$ is a real Banach space and $V$ is finite dimensional. Suppose $I \in C^{1}(E, R)$ is an even functional satisfying $I(0)=0$ and

(II) there is a constant $\rho>0$ such that $\left.I\right|_{\partial B_{\rho} \cap X} \geq 0$;

$\left(I_{2}\right)$ there is a subspace $W$ of $E$ with $\operatorname{dim} V<\operatorname{dim} W<\infty$ and there is $M>0$ such that $\max _{u \in W} I(u)<M$;

$\left(I_{3}\right)$ considering $M>0$ given by $\left(I_{2}\right)$, I satisfies $(P S)_{c}$ for $0 \leq c \leq M$.

Then I possesses at least $\operatorname{dim} W-\operatorname{dim} V$ pairs of nontrivial critical points.

Using the version of the symmetric mountain pass theorem mentioned above, we can state our result as follows.

Theorem 2.1 Assume that $f(x, t)$ is odd in $t$, satisfies $\left(f_{*}\right)$ with $p^{-}<h^{+} \leq p^{+}$and the following assumptions:

$\left(f_{1}\right)$ there exist $\eta>p^{+}$and $1<\sigma<p^{-}$, and $a_{1}, a_{2}>0$, such that $\frac{1}{\eta} f(x, t) t-F(x, t) \geq-a_{1}-$ $a_{2}|t|^{\sigma}$ for every $t \in R$, a.e. in $\Omega$.

$\left(f_{2}\right)$ there is $\Omega_{0} \subset \Omega$ with $\left|\Omega_{0}\right|>0$ such that $\liminf _{|t| \rightarrow \infty} F(x, t) /|t|^{p^{+}}=\infty$ uniformly a.e. in $\Omega_{0}$.

Then for any given $k \in N$, the problem (1.1) possesses at least $k$ pairs of nontrivial solutions.

\section{Main results and proofs}

In this section, we assume that $N \geq 1$ and $E=W_{0}^{1, P}(\Omega), u \in E$ is called a weak solution of the problem (1.1) if

$$
\int_{\Omega} a(|\nabla u|) \nabla u \nabla \phi d x=\int_{\Omega} f(x, u) \phi d x, \quad \forall \phi \in E .
$$

Set

$$
I(u)=\int_{\Omega} P(|\nabla u|) d x-\int_{\Omega} F(x, u) d x, \quad \forall u \in E
$$

and we know that the critical points of $I$ are just the weak solutions of the problem (1.1).

For $E$ is a separable and reflexive Banach space, then there exist (see [9]) $\left\{e_{n}\right\}_{n=1}^{\infty} \subset E$ and $\left\{e_{n}^{*}\right\}_{n=1}^{\infty} \subset E^{*}$ such that

$$
e_{n}^{*}\left(e_{m}\right)=\delta_{n, m}=\left\{\begin{array}{ll}
1, & \text { if } n=m ; \\
0, & \text { if } n \neq m .
\end{array} \quad \text { and } \quad e_{n}^{*}(v)=\alpha_{n} \quad \text { for } v=\sum_{i=1}^{\infty} \alpha_{i} e_{i} \in E\right.
$$

Now, we set $V_{j}=\left\{u \in W_{0}^{1, P}(\Omega): e_{i}^{*}(u)=0, i>j\right\}, X_{j}=\left\{u \in W_{0}^{1, P}(\Omega): e_{i}^{*}(u)=0, i \leq j\right\}$, so

$$
W_{0}^{1, P}(\Omega)=V_{j} \oplus X_{j}
$$


Lemma 3.1 Given $\delta>0$, there is $j \in N$ such that for all $u \in X_{j},|u|_{H} \leq \delta\|u\|$.

Proof We prove the lemma by contradiction. Suppose that there exist $\delta>0$ and $u_{j} \in X_{j}$ for every $j \in N$ such that $\left|u_{j}\right|_{H} \geq \delta\left\|u_{j}\right\|$. Taking $v_{j}=\frac{u_{j}}{\left|u_{j}\right| H}$, we have $\left|v_{j}\right|_{H}=1$ for every $j \in N$ and $\left\|v_{j}\right\| \leq \frac{1}{\delta}$. Hence, $\left\{v_{j}\right\} \subset W_{0}^{1, P}(\Omega)$ is a bounded sequence, and we may suppose, without loss of generality, that $v_{j} \rightarrow v$ in $W_{0}^{1, P}(\Omega)$. Furthermore, $e_{n}^{*}(v)=0$ for every $n \in N$ since $e_{n}^{*}\left(v_{j}\right)=0$ for all $j \geq n$. This shows that $v=0$. On the other hand, by the compactness of embedding $W_{0}^{1, P}(\Omega) \hookrightarrow L^{H}(\Omega)$, we conclude that $|v|_{H}=1$. This proves the lemma.

Lemma 3.2 Suppose $f$ satisfies $\left(f_{*}\right)$, then there exist $j \in N$ and $\rho, \alpha>0$ such that

$$
\left.I\right|_{\partial B_{\rho} \cap X_{j}} \geq \alpha .
$$

Proof Now suppose that $\|u\|>1$. From $\left(f_{*}\right)$, we know that

$$
\begin{aligned}
I(u) & =\int_{\Omega} P(|\nabla u|) d x-\int_{\Omega} F(x, u) d x \\
& \geq\|u\|^{p^{-}}-C_{1}|u|_{H}^{h^{+}}-C_{2} .
\end{aligned}
$$

Consequently, considering $\delta>0$ to be chosen posteriorly by Lemma 3.1, we have for all $u \in X_{j}$ and $j$ sufficiently large,

$$
I(u) \geq\|u\|^{p^{-}}\left(1-C_{1} \delta^{h^{+}}\|u\|^{h^{+}-p^{-}}\right)-C_{2} .
$$

Now, taking $\|u\|=\rho(\delta)=\left(\frac{1}{2 C \delta^{h^{+}}}\right)^{\frac{1}{h^{+}-p^{-}}}$and noting that $\rho(\delta) \rightarrow+\infty$, if $\delta \rightarrow 0$, we can choose $\delta>0$ such that $\frac{1}{2} \rho^{p^{-}}>C_{2}, \rho>1$, and $I(u)>0$ for every $u \in X_{j}$, $\|u\|=\rho$, the proof is complete.

Lemma 3.3 Supposef satisfies $\left(f_{2}\right)$. Then given $m \in N$, there exist a subspace $W$ of $W_{0}^{1, P}(\Omega)$ and a constant $M_{m}>0$ such that $\operatorname{dim} W=m$ and $\max _{u \in W} I(u)<M_{m}$.

Proof Let $x_{0} \in \Omega_{0}$ and $r_{0}>0$ be such that $\overline{B\left(x_{0}, r_{0}\right)} \subset \Omega$, and $0<\left|\overline{B\left(x_{0}, r_{0}\right)} \cap \Omega_{0}\right|<\frac{\left|\Omega_{0}\right|}{2}$. First, we take $v_{1} \in C_{0}^{\infty}(\Omega)$ with $\operatorname{supp}\left(v_{1}\right)=\overline{B\left(x_{0}, r_{0}\right)}$. Considering $\Omega_{1}=\Omega_{0} \backslash\left[\overline{B\left(x_{0}, r_{0}\right)} \cap\right.$ $\left.\Omega_{0}\right] \subset \widehat{\Omega}_{0}=\overline{\Omega \backslash B\left(x_{0}, r_{0}\right)}$, we have $\left|\Omega_{1}\right| \geq \frac{\left|\Omega_{0}\right|}{2}>0$. Let $x_{1} \in \Omega_{1}$ and $r_{1}>0$ be such that $\overline{B\left(x_{1}, r_{1}\right)} \subset \widehat{\Omega}_{0}$, and $0<\left|\overline{B\left(x_{1}, r_{1}\right)} \cap \Omega_{1}\right|<\frac{\left|\Omega_{1}\right|}{2}$. Next, we take $v_{2} \in C_{0}^{\infty}(\Omega)$ with $\operatorname{supp}\left(v_{2}\right)=$ $\overline{B\left(x_{1}, r_{1}\right)}$. After a finite number of steps, we get $v_{1}, v_{2}, \ldots, v_{m}$ such that $\operatorname{supp}\left(v_{i}\right) \cap \operatorname{supp}\left(v_{j}\right)=\emptyset$, $i \neq j$, and $\left|\operatorname{supp}\left(v_{j}\right) \cap \Omega_{0}\right|>0$ for all $i, j \in\{1,2, \ldots, m\}$. Let $W=\operatorname{span}\left\{v_{1}, v_{2}, \ldots, v_{m}\right\}$, by construction, $\operatorname{dim} W=m$, and $\int_{\Omega}|\nu|^{p^{+}} d x>0$ for every $v \in W \backslash\{0\}$.

Since $\max _{u \in W \backslash\{0\}} I(u)=\max _{t>0, v \in W \cap \partial B_{1}(0)}\left(\int_{\Omega} P(t|\nabla v|) d x-\int_{\Omega} F(x, t v) d x\right)$, if $t>1$, then $I(t v) \leq t^{p^{+}}-\int_{\Omega} F(x, t v) d x=t^{p^{+}}\left(1-\frac{1}{t^{p^{+}}} \int_{\Omega} F(x, t v) d x\right)$. Now, it suffices to verify that

$$
\lim _{t \rightarrow \infty} \frac{1}{t^{p^{+}}} \int_{\Omega} F(x, t v) d x>1
$$

From the condition $\left(f_{2}\right)$, given $L>0$, there is $C>0$ such that for every $s \in R$, a.e. $x$ in $\Omega_{0}$,

$$
F(x, s) \geq L|s|^{p^{+}}-C .
$$


Consequently, for $v \in \partial B_{1}(0) \cap W$ and $t>1$,

$$
\int_{\Omega} F(x, t v) d x \geq L t^{p^{+}} \int_{\Omega_{0}}|v|^{p^{+}} d x-C t^{h^{+}} \int_{\Omega \backslash \Omega_{0}} H(v) d x-C_{2},
$$

and

$$
\lim _{t \rightarrow \infty} \frac{\int_{\Omega} f(x, t v) d x}{t^{p^{+}}} \geq L \int_{\Omega_{0}}|v|^{p^{+}} d x-C \int_{\Omega \backslash \Omega_{0}} H(v) d x \geq L r-C R,
$$

where $r=\min \left\{\int_{\Omega_{0}}|v|^{p^{+}} d x, v \in \partial B_{1}(0) \cap W\right\}$ and $R=\max \left\{\int_{\Omega \backslash \Omega_{0}} H(v) d x, v \in \partial B_{1}(0) \cap W\right\}$. Observing that $W$ is finite dimensional and we have $R<+\infty, r>0$, the inequality is obtained by taking $L>\frac{1}{r}(1+C R)$; the proof is complete.

Lemma 3.4 Suppose $f$ satisfies $\left(f_{1}\right)$, then I satisfies the (PS) condition.

Proof We suppose that $\left\|u_{n}\right\|>1$,

$$
\begin{aligned}
M & +o(1)\left\|u_{n}\right\| \\
& \geq I\left(u_{n}\right)-\frac{1}{\eta} I^{\prime}\left(u_{n}\right) u_{n} \\
& =\int_{\Omega} P\left(\left|\nabla u_{n}\right|\right) d x-\frac{1}{\eta} \int_{\Omega} p\left(\left|\nabla u_{n}\right|\right) \nabla u_{n} d x+\int_{\Omega}\left(\frac{1}{\eta} f\left(x, u_{n}\right) u_{n}-F\left(x, u_{n}\right)\right) d x \\
& \geq\left(1-\frac{p^{+}}{\eta}\right)\left\|u_{n}\right\|^{p^{-}}-a_{1}|\Omega|-C\left\|u_{n}\right\|^{\sigma} .
\end{aligned}
$$

Noting that $1<\sigma<p^{-}, \eta>p^{+},\left\{u_{n}\right\}$ is bounded. By [9], Lemma 3.1, we know that $I$ satisfies the (PS) condition.

Proof of Theorem 2.1 First, we recall that $W_{0}^{1, P}(\Omega)=V_{j} \oplus X_{j}$, where $V_{j}$ and $X_{j}$ are defined in (3.1). Invoking Lemma 3.2, we find $j \in N$, and $I$ satisfies $I_{1}$ with $X=X_{j}$. Now, by Lemma 3.3, there is a subspace $W$ of $W_{0}^{1, P}(\Omega)$ with $\operatorname{dim} W=k+j=k+\operatorname{dim} V_{j}$ and such that $I$ satisfies $\left(I_{2}\right)$. Since $I(0)=0$ and $I$ is even, we may apply Lemma 2.4 to conclude that $I$ possesses at least $k$ pairs of nontrivial critical points. The proof is complete.

\section{Competing interests}

The authors declare that they have no competing interests.

\section{Authors' contributions}

All authors read and approved the final manuscript.

\section{Author details}

'School of Science, Jiangnan University, Wuxi, 214122, People's Republic of China. ${ }^{2}$ School of Mathematics Science, Nanjing Normal University, Nanjing, 210097, People's Republic of China.

\section{Acknowledgements}

Project supported by Natural Science Foundation of China, Tian Yuan Special Foundation (No. 11226116), Natural Science Foundation of Jiangsu Province of China for Young Scholar (No. BK201209), the China Scholarship Council, the Fundamental Research Funds for the Central Universities (No. JUSRP11118) and Foundation for young teachers of Jiangnan University (No. 2008LQN008). 


\section{References}

1. Clément, PH, García-Huidobro, M, Manásevich, R, Schmitt, K: Mountain pass type solutions for quasilinear elliptic equations. Calc. Var. Partial Differ. Equ. 11, 33-62 (2000)

2. Fukagai, N, Ito, M, Narukawa, MK: Positive solutions of quasilinear elliptic equations with critical Orlicz-Sobolev nonlinearity on $R^{N}$. Funkc. Ekvacioj 49, 235-267 (2006)

3. Fukagai, N, Narukawa, K: On the existence of multiple positive solutions of quasilinear elliptic eigenvalue problems. Ann. Mat. Pura Appl. 186, 539-564 (2007)

4. García-Huidobro, M, Le, V, Manásevich, R, Schmitt, K: On the principal eigenvalues for quasilinear elliptic differential operators: an Orlicz-Sobolev space setting. Nonlinear Differ. Equ. Appl. 6, 207-225 (1999)

5. Tan, Z, Fang, F: Orlicz-Sobolev versus Hölder local minimizer and multiplicity results for quasilinear elliptic equations. Preprint

6. Mihǎilescu, M, Rădulescu, V: Nonhomogeneous Neumann problems in Orlicz-Sobolev spaces. C. R. Math. 346 401-406 (2008)

7. Bonanno, G, Bisci, GM, Rădulescu, VD: Quasilinear elliptic non-homogeneous Dirichlet problems through Orlicz-Sobolev spaces. Nonlinear Anal. 75, 4441-4456 (2012)

8. Černý, R: Generalized $n$-Laplacian: quasilinear nonhomogenous problem with critical growth. Nonlinear Anal. 74, 3419-3439 (2011)

9. Fang, F, Tan, Z: Existence and multiplicity of solutions for a class of quasilinear elliptic equations: an Orlicz-Sobolev space setting. J. Math. Anal. Appl. 389, 420-428 (2012)

10. Adams, RA, Fournier, JJF: Sobolev Spaces, 2nd edn. Academic Press, Amsterdam (2003)

11. Ambrosetti, A, Rabinowitz, PH: Dual variational methods in critical point theory and applications. J. Funct. Anal. 14, 349-381 (1973)

12. Bartolo, P, Benci, V, Fortunato, D: Abstract critical point theorems and applications to some nonlinear problems with "strong" resonance at infinity. Nonlinear Anal. TMA 7, 981-1012 (1983)

13. Silva, EAB: Critical point theorems and applications to differential equations. PhD thesis, University of Wisconsin-Madison (1988)

doi:10.1186/1687-2770-2012-136

Cite this article as: Yang and Zhang: A note on the existence of solutions for a class of quasilinear elliptic equations: an Orlicz-Sobolev space setting. Boundary Value Problems 2012 2012:136.

\section{Submit your manuscript to a SpringerOpen ${ }^{\circ}$ journal and benefit from:}

- Convenient online submission

- Rigorous peer review

- Immediate publication on acceptance

- Open access: articles freely available online

High visibility within the field

Retaining the copyright to your article 